\title{
Comparison of Two Autonomous AC-DC Converters for Piezoelectric Energy Scavenging Systems
}

\author{
Enrico Dallago ${ }^{1}$, Daniele Miatton ${ }^{1}$, Giuseppe Venchi ${ }^{1}$, Valeria Bottarel ${ }^{2}$, \\ Giovanni Frattini ${ }^{2}$, Giulio Ricotti ${ }^{2}$, and Monica Schipani ${ }^{2}$ \\ ${ }^{1}$ Department of Electrical Engineering, University of Pavia, 27100 Pavia, Italy \\ ${ }^{2}$ STMicroelectronics 20010 Cornaredo, Milan, Italy
}

\begin{abstract}
Piezoelectric Energy Scavenging Systems (PESS) are used to convert the energy of mechanical vibrations into electrical energy exploiting the piezoelectric effect. Their output is a voltage which strongly varies in time; to obtain a suitable supply source an AC-DC conversion of the output voltage of these transducers is needed. Since the output power level of the energy transducer can be very low, the conversion should be as efficient as possible.

The paper describes an active AC-DC converter, based on the voltage doubler topology, in which two different driving circuitries have been implemented.

The proposed solutions are fully autonomous, i.e. they are supplied by the energy that they harvest. To reduce and to optimize their power consumption a bias circuit has been designed to make the total bias current supply independent.

A test chip has been diffused using STMicroelectronics 5V CMOS technology. The performances of the two solutions were compared with the ones of a passive Schottky based voltage doubler. The figures of merit were the average power supplied by the piezoelectric transducer and the average power delivered on a load resistance. Furthermore, the significance of such parameters is also discussed, and a more general figure of merit is defined with the advantage of also weighting the ability of the converter to harvest all the power available at the transducer terminals.
\end{abstract}

Keywords: Piezoelectric energy harvesting, active AC-DC converter, low-power circuits, environmental vibrations.

\section{Introduction}

Energy scavenging systems are used to harvest the normally lost environmental energy (associated to vibrations, thermal gradients, solar radiation, pressure gradients) and to convert it into electrical energy. This solution can be very attractive to supply portable or low power electronic devices where batteries are a bottleneck for the whole system (i.e. they have a finite life time and their replacement or recharge is not feasible or too expensive). An energy scavenging system, instead, is a theoretically endless energy source. For this reason they are rapidly gaining popularity in the scientific and the industrial community. Typical applications are wireless sensing (both single spot and multi-nodes systems), biomedical (patient monitoring, hearing aids, etc.), automotive (using self supplied, wireless devices can help reducing the 
cable length on board). The challenge is twofold: on one side the power requirements of the supplied electronics should be as low as possible, on the other, given the very low energetic content associated to environmental energy, the interface circuit has to be as effective as possible in managing the harvested power.

In literature many papers can be found which describe methodologies to realize an energy-scavenger [1], [6], [8], [10]. Each case has different requirements depending on the environmental energy source considered, the characteristics of the transducer, the typical frequency of the process. No universal solution exists: each scavenger interface electronics has to be tailored to the specific transducer and application.

Many of the above mentioned works are focused on the conversion of the energy associated to mechanical vibrations since they can be easily found in many environments [1], [7]. Among the available vibration transducers this paper considers a system based on a piezoelectric transducer since it is one of the most efficient which can be used [1]-[2]. When exposed to vibrations coming from the real world the electrical energy at the output of this transducer is a strong and irregular function of time [1]-[4], [9], hence, to realize a DC supply source, an AC-DC conversion is needed.

Two active AC-DC converters based on the voltage doubler topology (see Figure 1) are presented. The reasons behind the choice of this topology are the fact that is it simple and that it is able to increase the value of the input voltage by approximately a factor two. This last aspect is quite important: as it will be shown later, the piezoelectric transducer exhibits a resonant behaviour; at resonance the output voltage for a given acceleration can be quite high, but out of the resonance the same acceleration will produce a voltage much lower. In perspective of an actual application, the harvester will be exposed to an acceleration whose spectrum is spread over a range, rather than being tuned at a particular frequency, so being able to stepup the input voltage is a desired feature.

The idea of implementing an active rectifier, rather than a passive one, is basically inspired by the observation that the voltage drop across the switches of the rectifier causes power dissipation and, most of all, has to be subtracted from the output

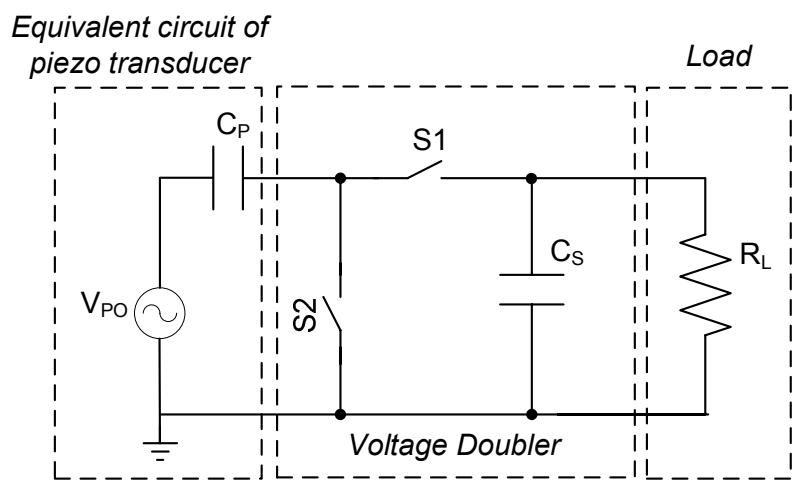

Fig. 1. Piezoelectric energy harvesting system 
voltage, decreasing it and consequently the energy that can be stored at the output (for example across a capacitor as shown in Fig. 1). Of course the active circuitry comes at the price of using a part of the harvested energy to supply the active circuitry itself: active converters can be more efficient than passive solution [3-4], [11], but they are more difficult to be designed because their whole power consumption has to be very low.

To this purpose, and for other reasons which will be illustrated in the following, two different driving circuitries were considered to command switches S1 and S2 (Figure 2). In particular, the topology shown in Figure 2a, which will be called "hard", uses comparators (CMP1-2), while in the one of Figure 2b, which will be called "soft", the switches are driven with operational amplifiers (OP1-2). Both proposed solutions use only a fraction of the harvested energy to supply the active circuitry and make the energy scavenging system autonomous. In particular, at the output a capacitance $\left(\mathrm{C}_{\mathrm{S}}\right)$ of $1 \mu \mathrm{F}$ is used to store the harvested energy and no external power source is required, neither during start-up nor in normal operation ${ }^{1}$.

Since the voltage across the storage capacitor Cs increases as the energy is harvested, a supply independent bias circuitry is used in both cases: it makes the current consumption of the active part supply independent.

Both circuits were diffused as test chips exploiting a 5V CMOS STMicroelectronics technology and were extensively tested. They were compared in terms of efficiency of the converter and of the average output power, evaluated under the same input conditions while varying the load. The significance of such parameters is also discussed, and a more general figure of merit is defined with the advantage of also weighting the ability of the converter to harvest all the power available at the transducer terminals.

Section 2 deals with the design of the two considered AC-DC driving circuitries highlighting their pro and cons, while Section 3 shows the experimental results compared with the simulated ones.

\section{Piezoelectric Energy Scavenging System}

\subsection{Mechanical Aspects}

The considered piezoelectric transducer is a cantilever which works in 31-mode when it is excited by the mechanical vibrations, as shown in Figure 3. This means that the cantilever is exposed to a strain in the direction of axis 1 while the resulting electrical displacement is along axis 3 .

To have a maximally efficient conversion of the mechanical vibrations into electrical energy the cantilever should be excited at its resonant frequency which can

\footnotetext{
${ }^{1}$ The presence of the output capacitor is not only required to supply the control circuitry or to filter out the voltage ripple, but it often works as an energy tank. In fact, in most cases the supplied electronics works with a duty cycle. For example a wireless sensor that transmits one measurement every 10 seconds will be active only for a few hundreds of milliseconds, while it will switch in a low consumption or standby mode for the rest of the time. During this interval the scavenger is expected to accumulate the energy required during the burst of operation.
} 

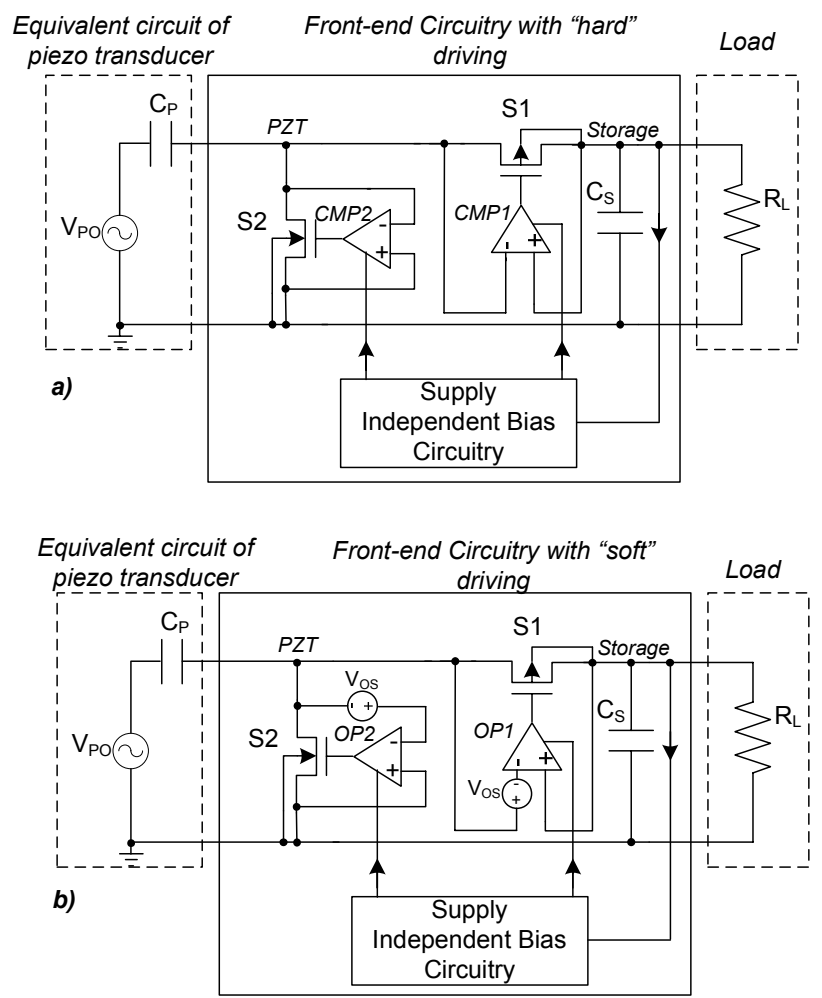

Fig. 2. Schematics of the proposed ESS. Voltage doubler with "hard" driving circuitry (a) and with "soft" driving circuitry (b).

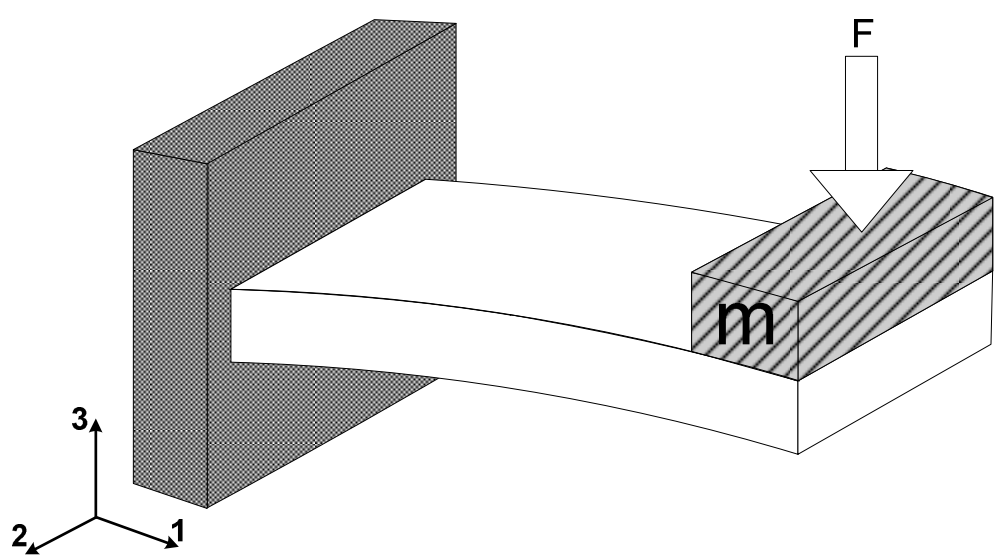

Fig. 3. Piezoelectric energy transducer working in 31-mode 
be varied adding a mass $(\mathrm{m})$ on its free end [1]. This allows to move the resonance frequency of the energy transducer around the frequency which is more likely in the application. The experimental measurements shown in Table 1 and [1] show that the frequency range of mechanical vibrations existing into civil environments is approximately (10-380) $\mathrm{Hz}$.

Table 1. Experimental measurements of environmental vibration sources

\begin{tabular}{|c|c|c|}
\hline Environmental Vibration Source & $\mathbf{a}_{\mathbf{r m s}}\left[\mathbf{m} / \mathbf{s}^{\mathbf{2}}\right]$ & Bandwidth [Hz] \\
\hline CD reader of a desktop PC & 0,30 & 80 \\
\hline Pocket of a walking man & 2,16 & 2 \\
\hline Man walking onto stairs & 3,53 & 2 \\
\hline Into a woman bag & 6,37 & 10 \\
\hline Vibracall of a mobile phone & 3,83 & 90 \\
\hline Car dashboard [city street @ 20km/h] & 0,78 & 30 \\
\hline Car dashboard tortuous street @ 50km/h] & 0,88 & 20 \\
\hline Car front glass [primary street @ 50 & 0,98 & 15 \\
\hline km/h] & 0,10 & 50 \\
\hline Guard rail near a busy street & 0,10 & 100 \\
\hline Bridge over a railway & 0,19 & 10 \\
\hline Bus stop near a busy street & 1,40 & 100 \\
\hline Fun & 0,20 & 12 \\
\hline Web server & & \\
\hline
\end{tabular}

The piezoelectric transducer can be modeled at resonance by the equivalent circuit shown in Figure 1, [1]-[2]. Generator $\mathrm{V}_{\mathrm{PO}}$ is a sinusoidal voltage source whose frequency is equal to the transducer resonance frequency and whose amplitude is equal to the open circuit output voltage, while $C_{P}$ is the electrical capacitance of the piezoelectric cantilever.

The presented results are based on a piezoelectric transducer with geometrical dimensions equal to $(30 \times 15 \times 0.2) \mathrm{mm}^{3}(\mathrm{LxW} \times \mathrm{H})$. The piezoelectric material used was a soft Lead Titanate Zirconate (PZT) and it was sputtered onto a Nichel alloy support. The piezoelectric capacitance $\mathrm{C}_{\mathrm{P}}$ was measured to be $19 \mathrm{nF}$.

Figure 4 shows the experimental set-up which was used to shake the piezoelectric transducer with a controlled acceleration. It is composed of an electrodynamic shaker (Bruel\&Kjiaer 4810), which moves the piezoelectric transducer, and by a triaxial MEMS accelerometer (LIS3L02AS4 of STMicroelectronics); it was screwed to the shaker movable table so to measure the acceleration given by the shaker itself and to guarantee the repeatability of the measurements.

Figure 5 shows the experimental frequency response of the piezoelectric transducer when it was excited with a sinusoidal acceleration with a peak to peak amplitude 
equal to $0.8 \mathrm{~g}$. It is possible to see that its resonance frequency is equal about to $130 \mathrm{~Hz}$.

For example, the measured resonance frequency of the cantilever is about $64 \mathrm{~Hz}$ if a lead mass of about 0.8 grams is added.
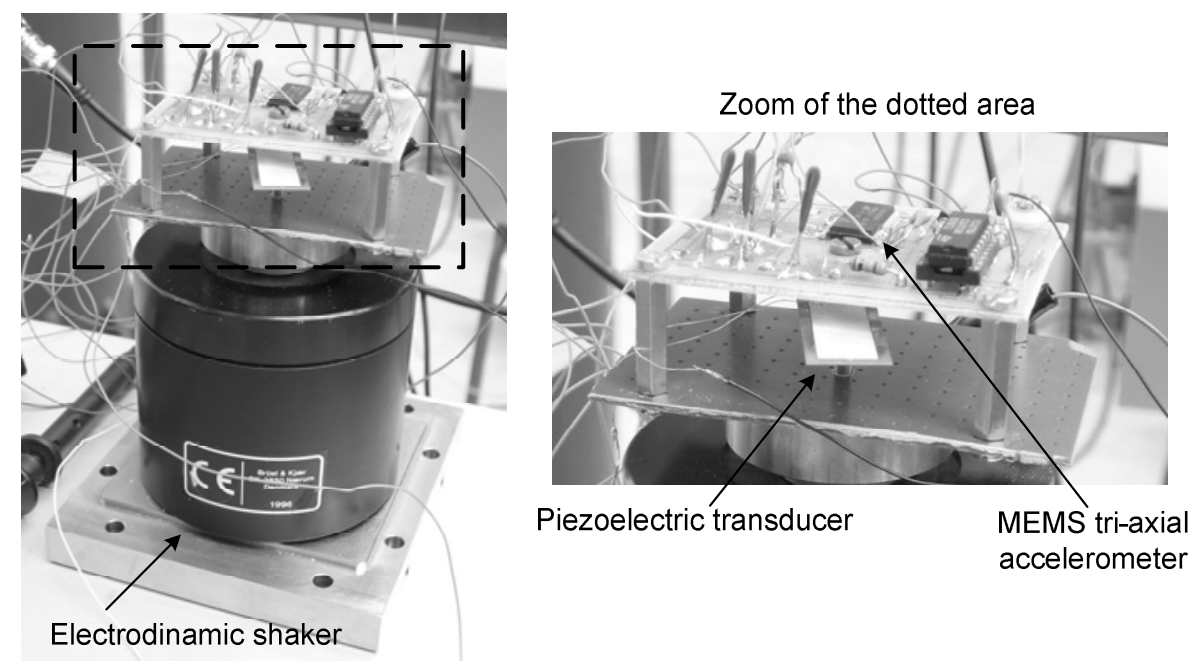

Fig. 4. Picture of the measurement set-up

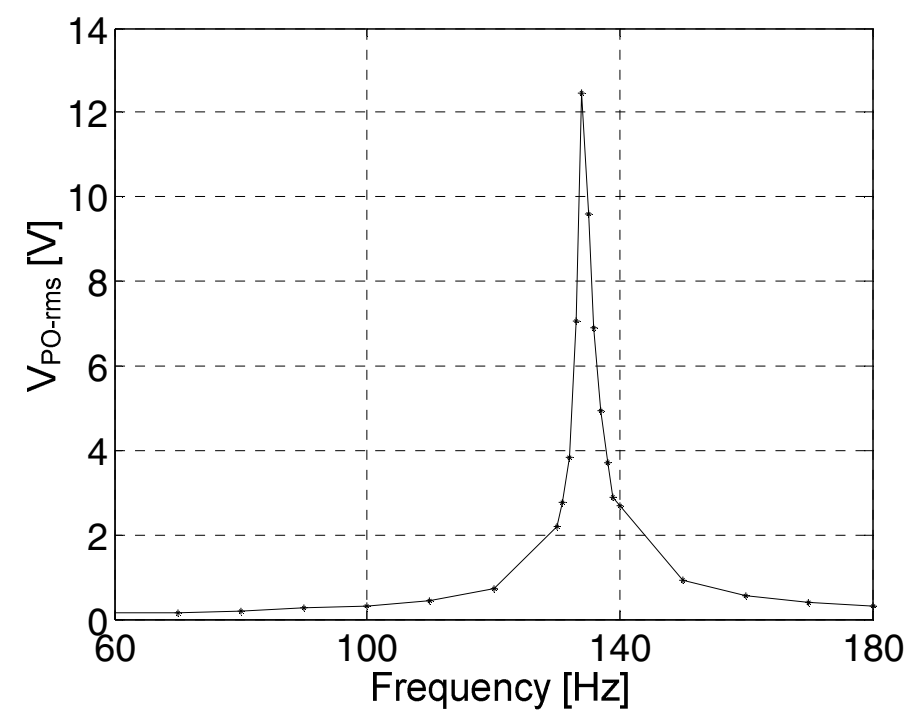

Fig. 5. Frequency response of the piezoelectric transducer 


\subsection{Design of the Proposed Front-End Circuitries: Common Parts}

During the start-up the voltage across $\mathrm{C}_{\mathrm{S}}$ is too low to supply the active devices. In this case, for both solutions, the operation of the converter is guaranteed by the body-drain diode of MOSFETs S1 and S2 which implement a standard, passive diode voltage doubler rectifier. Hence, the proposed rectifiers can be seen as a parallel of two AC-DC converters: an high efficiency active one and a lower efficiency passive one, the latter working only during start-up. As soon as the output voltage is sufficient the active one comes alive and flawlessly takes over the passive one; this switch does not need any dedicated control circuitry.

Furthermore, after the active part starts working, the voltage across $\mathrm{C}_{\mathrm{S}}$ varies with time as the energy is harvested. Since this voltage is used as the supply for the active devices, it is advantageous to make their current consumption independent on the supply voltage itself.

Figure 6 shows the circuital topology used to implement a supply independent bias; it is modified with respect to [5] by introducing a diode connected MOSFET (M4). In fact, since the system requires supply currents in the range of tens of nanoamperes, in the scheme without M4 the resistance R1 would be in the order of tens of megaohms, which is too area expensive for an integrated solution. The effect of M4 is to reduce the voltage drop across R1 lowering its value for a given current. Furthermore, a start up circuit is needed. This was obtained with dummy MOSFETs ML1 and ML2: the leakage of their body-drain diode has been exploited to inject a current into nodes A and $\mathrm{B}$ so to have the start-up aid. This solution allows us to avoid additional start-up circuitry, reducing total power consumption.

Figure 7 shows the simulated behaviour of the two bias voltages versus the supply voltage. In particular the circuitry starts to regulate when the supply voltage is higher than about $680 \mathrm{mV}$. Above this value, the voltages $\mathrm{V}_{\text {BiasP }}$ and $\mathrm{V}_{\text {BiasN }}$ can be used to mirror a supply independent current.

The current consumption of the whole bias circuitry is about 100nA.

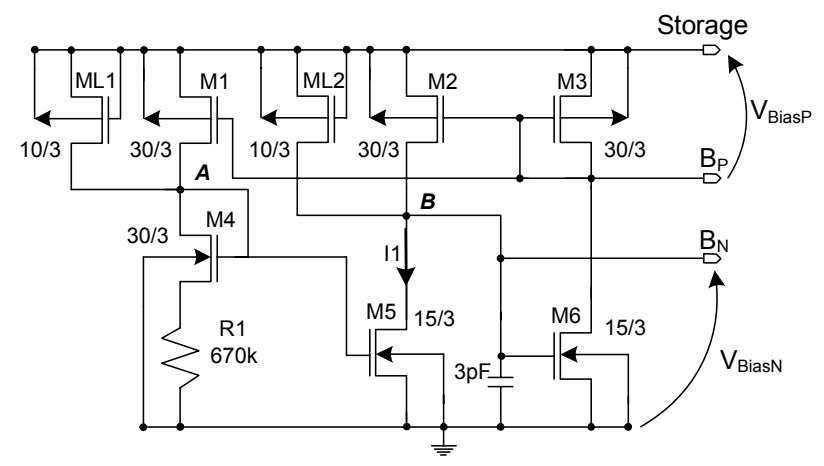

Fig. 6. Schematic of the proposed supply independent bias circuitry 


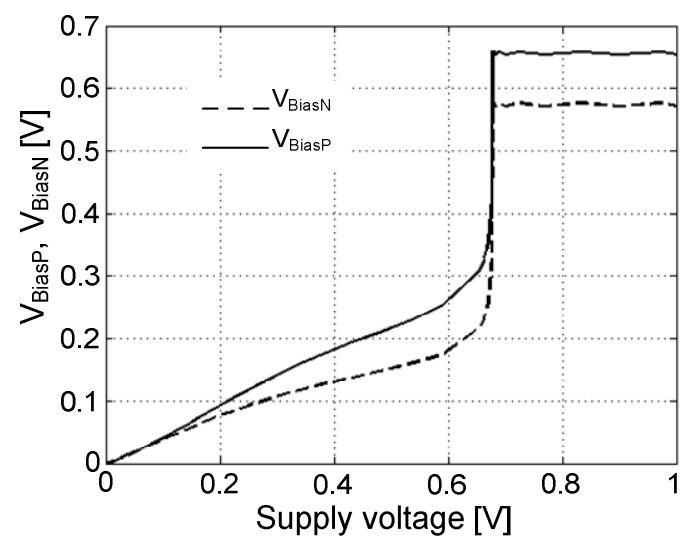

Fig. 7. Simulation results of the supply independent bias circuitry, bias voltages $\left(\mathrm{V}_{\mathrm{BiasN}}\right.$ and $\mathrm{V}_{\text {BiasP }}$ ) versus supply voltage (in the figure supply voltage has been limited to $1 \mathrm{~V}$ for clarity. The behaviour has been verified for supply voltages up to $5 \mathrm{~V}$ ).

\subsection{Working Principle of "Hard" Driving and Design Methodology}

Figure $2 \mathrm{a}$ shows the active topology of the ac-dc converter that uses comparators CMP1 and CMP2 to drive switches S1 and S2. The comparators sense the drain to source voltages of the two MOSFETs and properly turn them on or off [11]. This is the most straightforward way to implement an active version of the voltage doubler converter, since the switches and their driving emulate some ideal diode with a very low, almost zero, forward voltage drop, but the design of the two comparators is critical because a very accurate choice of the threshold voltages is required. In principle one could use a single threshold for each comparator equal to zero; in practice the spread due to process mismatches has to be taken into account. In fact if this voltage varies, during the diffusion process, with respect to the ideal value a negative effect, (i.e. the oscillation of the driving signal), can take place [4]. To reduce the probability to fall into this case the comparators has be designed with an hysteretic transfer characteristic, with an hysteresis wide enough to accommodate the expected variance of threshold. Among the two, the most critical threshold voltage, for both comparators, is the one which switches S1 or S2 off, called "Th-OFF". To prevent the above mentioned oscillations the designer has to guarantee it is positive for CMP1 and negative for CMP2; but, at the same time, to minimize the unwanted discharge of the piezoelectric or of the storage capacitance caused by a delayed switching [4], the "Th-OFF" should be as small as possible. These two requirements are, of course, contrasting.

Figure $8 \mathrm{a}$ and Figure $8 \mathrm{~b}$ show the electrical schemes of the two designed comparators that are supply and ground compatible respectively, while Figure $8 \mathrm{c}$ shows a picture of the diffused circuit. The bias voltages $B_{N}$ and $B_{P}$ are provided by 
the supply independent bias circuitry. Referring, for example, to CMP1 (similar considerations apply to CMP2) it is possible to see that the current in $\mathrm{M}_{4}$ depends on the output voltage of CMP1 itself. This allows to obtain the hysteretic transfer function for the comparator. In fact, when output voltage of CMP1 is low, (S1 is into its ON condition) the current in M4 is equal to the current in M5; on the contrary, when the output of CMP1 is high (S1 in OFF condition), an extra current, given by M3, flows in M4. As a consequence, the two threshold voltages are different from each other. To obtain a positive "Th-OFF" threshold voltage, a mismatch between the aspect ratio of the MOSFET M4 and M5 was introduced. In order to assure that the two "Th-OFF" voltages have the correct sign, 300 MonteCarlo simulations have been performed. The resulting distribution diagrams of the critical "Th-OFF" threshold voltages are shown in Figure 9 for both comparators. The chosen values are $+9 \mathrm{mV}$ and $-9 \mathrm{mV}$ for CMP1 and CMP2 respectively.

The static current consumption of each comparator is about $110 \mathrm{nA}$ and their minimum operating supply voltage is about $650 \mathrm{mV}$.

The structures indicated with "OD" into Figure 8c are two common source configuration MOSFETs whose gate terminals are connected to the output node of CMP1 and CMP2 respectively and whose drain terminals are directly connected to two output pads. These MOSFETs were added to allow the comparator output voltages to be measured, so to verify their functionality, without adding a loading capacitance too high to their output nodes.

\subsection{Working Principle of "Soft" Driving and Design Methodology}

Figure $2 \mathrm{~b}$ shows the active topology of the voltage doubler converter where the driving circuitry is realized with operational amplifiers OP1 and OP2.

To introduce its working principle let us consider Figure 10 which shows the case of OP1. It is possible to apply Kirchhoff Voltage Law (KVL) to the external mesh:

$$
V_{I N}+V_{S D}-V_{O S}=0
$$

If the operational amplifier has a DC gain equal to $A$ the voltage on the gate of $\mathrm{S} 1$ is:

$$
V_{G}=A \cdot V_{I N}
$$

Equations (1) and (2) can be solved as a function of $V_{G}$ :

$$
V_{G}=A \cdot\left(V_{O S}-V_{S D}\right)
$$

In the ideal case the DC gain $A$ of the operational amplifier is infinite; as a consequence the difference in equation (3) has to vanish in order to have a finite value of the gate voltage. Thus a regulation loop modulates the gate voltage $V_{G}$ so to keep, for each value of the drain current, the source to drain voltage is equal to Vos. 


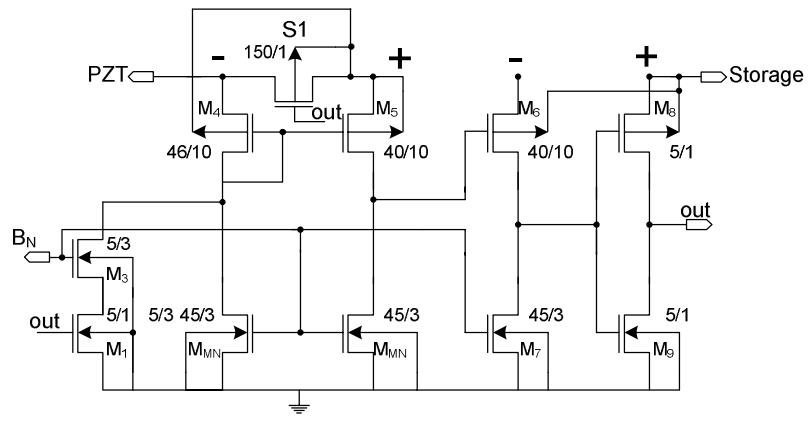

a)

b)
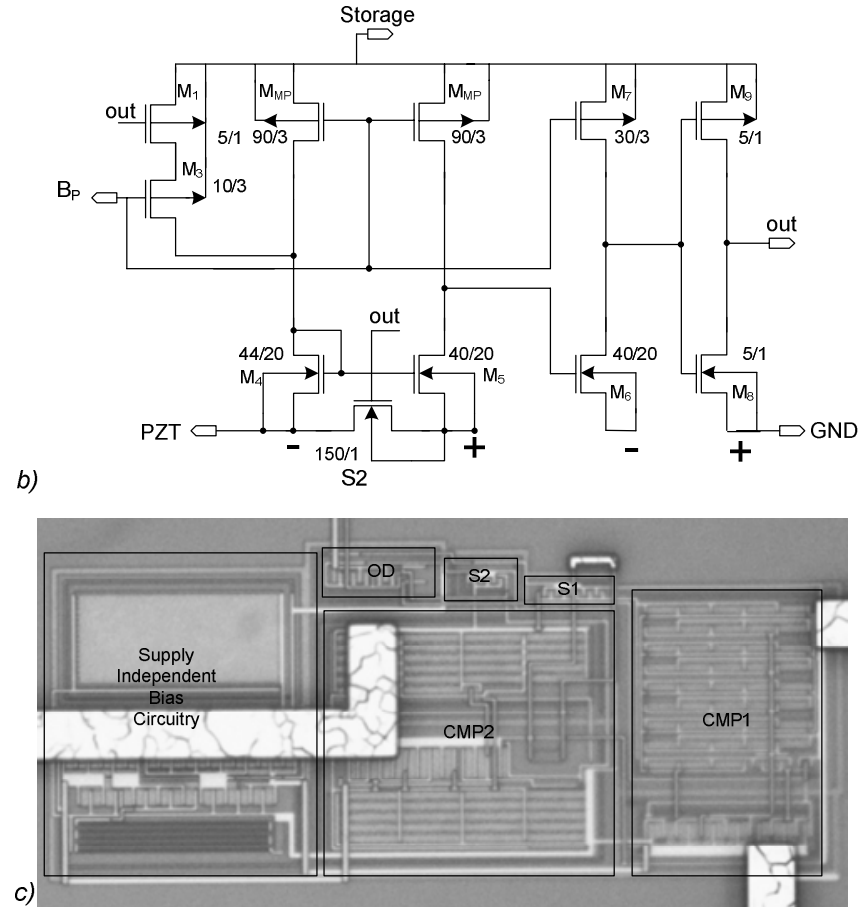

Fig. 8. a), b) Designed circuital topologies of CMP1 and CMP2 respectively; c) picture of the diffused "hard" AC-DC converter
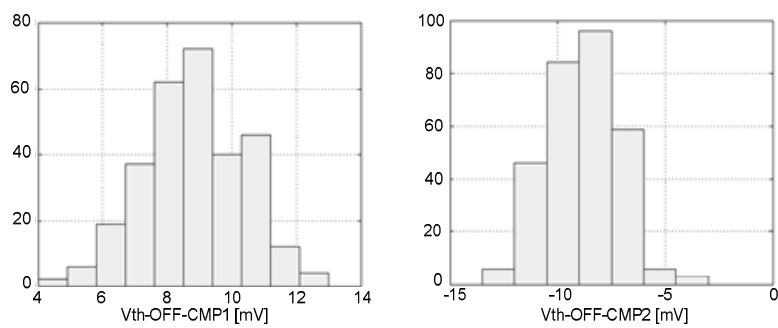

Fig. 9. MonteCarlo simulations of the most critical threshold voltage of the designed comparators 


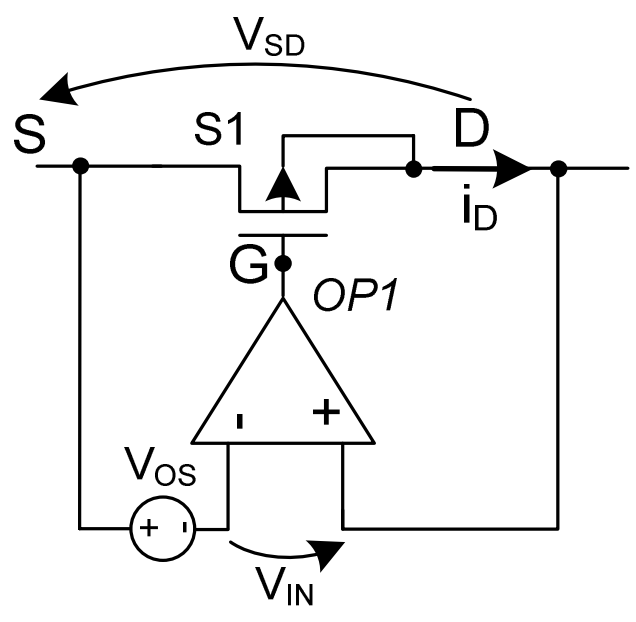

Fig. 10. Regulation loop composed by the operational amplifier OP1 and MOSFET S1

In the ideal case, when drain current is positive the regulation loop sets the working point of the MOSFET at the intersection between its characteristics and the offset voltage. When the current decreases, the loop moves the working point of the MOSFET at lower values of its source to gate voltage, until the current is equal to zero. At this point the regulation loop turns the MOSFET off: since there is no intersection between the MOSFET characteristics and the offset voltage, negative values of the current are not allowed and the regulation loop holds the transistor off. This principle intrinsically guarantees that, differently from the "hard" topology, no oscillations of the driving signal can take place. Furthermore, a negative current is impeded and the discharge of the capacitances is prevented. Symmetrical considerations apply to OP2. Differently from the "hard" approach, the value of Vos is not critical because it has simply to be far enough from zero so that the process mismatches will not change its sign. While it is true that an higher offset gives higher losses on the switch, they are still negligible for practical offset values (which can be easily designed in the tens of millivolts range).

In practice, the real operational amplifier has a finite DC gain; this means that the value of the voltage across the MOSFET is slightly different from the theoretical one. Nevertheless it can be demonstrated that, with the previously described choice of the offset, this will not affect the operation of the circuit.

Figure 11a shows the designed operational amplifiers while Figure 11b contains a picture of the diffused structure. Because of the level of their input voltages, OP1 and OP2 have to be supply compatible and ground compatible respectively.

The bias of the operational amplifiers is given by the supply independent bias circuitry. The operational amplifiers have been designed so that they are able to work with the minimum possible value of the voltage supply. In this way the active rectifier takes over the passive one as soon as a very low energy has been stored into capacitance $\mathrm{C}_{\mathrm{S}}$. A $5 \mathrm{pF}$ capacitance has been introduced to compensate the regulation loop. 
The offset voltage was obtained mismatching the aspect ratio of the input MOSFETs $M_{A}$ and $M_{B}$ : the values of the obtained offset voltages are equal to $26 \mathrm{mV}$ and $21 \mathrm{mV}$ for OP1 and OP2 respectively. Figure 12 presents the simulation results of 500 MonteCarlo iterations, showing the possible spread of these voltages. It is possible to see that this spread is sufficiently small to have the correct sign also with process mismatches.

Aspect ratio of the MOSFETs S1 and S2 has to be chosen to avoid the loop saturation. The expression of the drain current when the MOSFET is turned on is, in a first approximation, equal to:

$$
i_{D}=k \frac{W}{L}\left(V_{G S}-V_{t h}\right) V_{D S}
$$

where $\mathrm{V}_{\text {th }}$ is the threshold voltage of the MOSFET, W/L is its aspect ratio and $\mathrm{k}$ is its characteristic constant.

The term into the parenthesis is the overdrive voltage: its value is modulated by the regulation loop which, for each drain current, varies the gate voltage.

If the overdrive voltage is enough the source to drain voltage is equal to $\mathrm{V}_{\mathrm{OS}}$, that is:

$$
i_{D}=k \frac{W}{L}\left(V_{G S}-V_{t h}\right) V_{O S}
$$
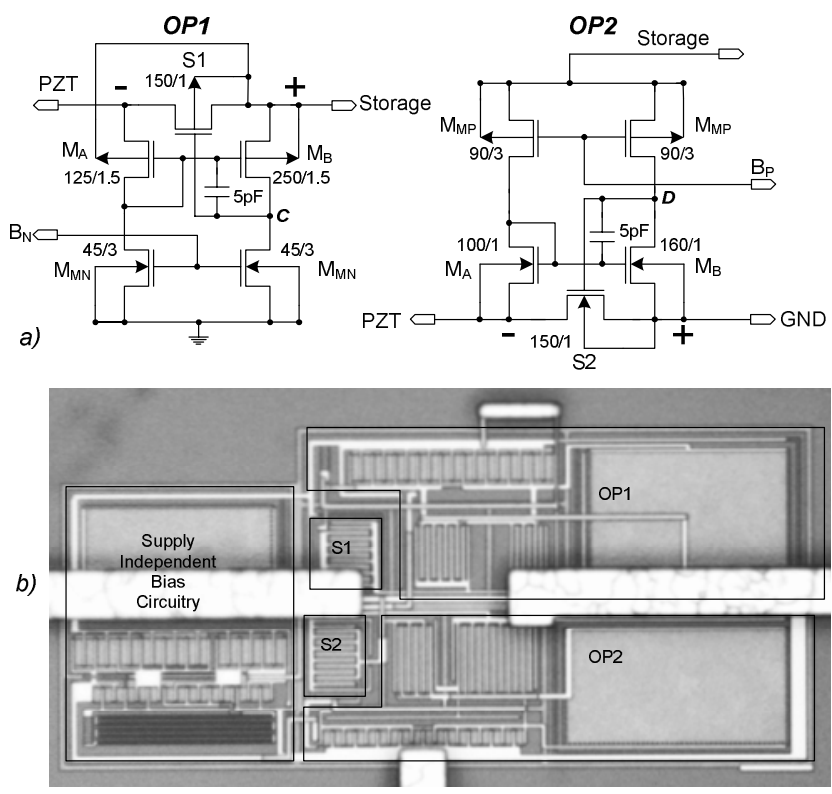

Fig. 11. a) Schematic of the proposed operational amplifiers; b) picture of the diffused "soft" AC-DC converter 

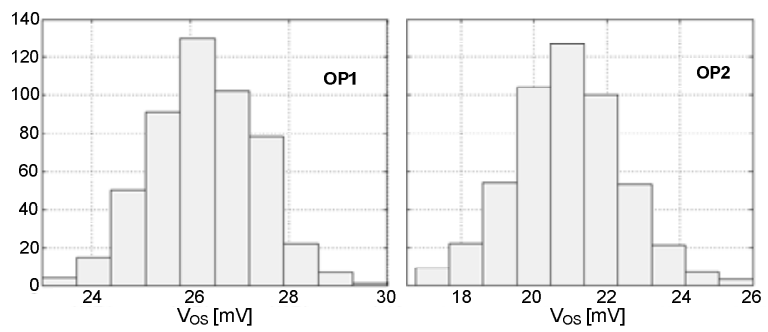

Fig. 12. MonteCarlo simulations of the offset voltages of the designed operational amplifiers

On the other hand if, for a given current, the overdrive is not enough (which means that the op-amp is saturated) the regulation loop does not work and the source to drain voltage of S1 or S2 is not equal to offset voltage.

The aspect ratio has to be designed to accommodate the maximum expected current.

Tab. 2 resumes the characteristics obtained for the amplifiers: in particular the current consumption is about $200 \mathrm{nA}$ for each amplifier. $\mathrm{V}_{\mathrm{dd}-\mathrm{min}}$ is the minimum supply voltage required by the operational amplifiers to work.

Table 2. Characteristics of the designed operational Amplifiers

\begin{tabular}{|l|c|c|c|c|c|}
\hline & $\begin{array}{c}\text { DC-GAIN } \\
{[\mathbf{d B}]}\end{array}$ & $\begin{array}{c}\text { Band-Width } \\
{[\mathbf{H z}]}\end{array}$ & $\begin{array}{c}\text { Vdd-min } \\
{[\mathbf{m V}]}\end{array}$ & $\begin{array}{c}\text { Voffset } \\
{[\mathbf{V}]}\end{array}$ & $\begin{array}{c}\text { Current Consumption } \\
{[\mathbf{n A}]}\end{array}$ \\
\hline $\boldsymbol{O P 1}$ & 50.37 & 2500 & 730 & $26 \mathrm{e}-3$ & 200 \\
\hline $\boldsymbol{O P 2}$ & 49.78 & 2610 & 675 & $21 \mathrm{e}-3$ & 200 \\
\hline
\end{tabular}

\section{Experimental and Simulated Results}

Before evaluating the performances of the proposed solutions an experimental characterization of the supply independent bias circuit was realized. This was done using a stand alone version of the circuit depicted in Figure 13b: three MOSFETs Ma, $\mathrm{Mb}$ and $\mathrm{Mc}$ were added to amplify the bias current by a factor 30 . This current is then converted into a voltage drop by means of an external resistance, equal to $2 \mathrm{M} \Omega$. Experimental characterization was done supplying the bias circuit with a triangular waveform in the range $0-5 \mathrm{~V}$. Figure $13 \mathrm{a}$ shows that, as soon as the supply voltage is higher than $680 \mathrm{mV}$, the circuit generates a constant voltage drop across the $2 \mathrm{M} \Omega$ resistance which corresponds to a constant bias current, as it was predicted by the simulations.

The performances of the proposed solutions have been evaluated at different values of the load resistance $\mathrm{R}_{\mathrm{L}}$; in particular, the considered figures of merit were the average power at the output of the piezoelectric transducer $\left(\mathrm{P}_{\mathrm{P}}\right)$ and the average power delivered to $R_{L}$ itself $\left(P_{L}\right)$. The load resistance was varied between $50 \mathrm{k} \Omega$ to $550 \mathrm{k} \Omega$. 
A passive voltage doubler, realized with BAT-86 Schottky diodes that have a threshold voltage equal to $0.2 \mathrm{~V}$, was considered as a benchmark. This comparison is quite significant since the selected diodes as a forward voltage drop way lower than a standard diode and the passive solution does not have any additional consumption due to the control circuitry.

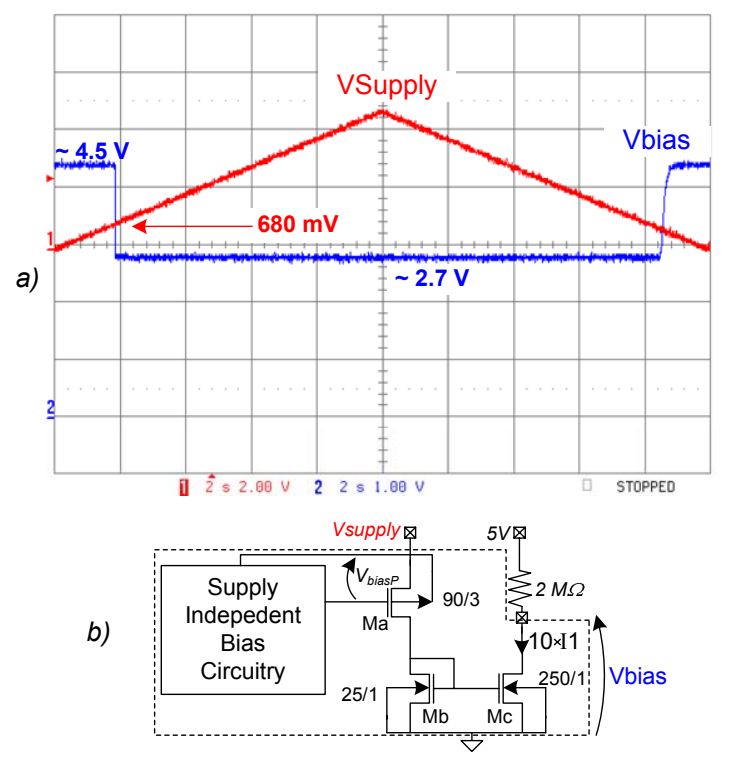

Fig. 13. a) Experimental results of the supply independent bias circuitry; b) circuitry used to do the characterization

Figure 14 compares the experimental results of the "hard" solution with those of the passive converter. The piezoelectric transducer was mechanically excited at $130 \mathrm{~Hz}$ and the acceleration was regulated so that the peak value of $\mathrm{V}_{\mathrm{PO}}$ was equal to $1.5 \mathrm{~V}$.

The powers drawn from the piezoelectric transducer are comparable between the two cases. On the contrary, the output power of the "hard" solution is heavily affected at low load resistance values. This is due to the fact that at values lower than $100 \mathrm{k} \Omega$ the energy stored into $\mathrm{C}_{\mathrm{S}}$, and hence the voltage across it, is not enough to switch the driving circuitry on and, as a consequence, only the well-source diodes of the passive path are exploited for the conversion. Conversely, at higher load values the converter works in the active way and the output power is higher with respect to the passive solution. This confirms that the lower forward voltage drop across the switches has positive effects on the output power, consistent enough to compensate for the power consumption of the control circuitry.

Furthermore, the accuracy of the design has guaranteed that, in spite of the fact S1 and S2 are switched off with a delay due to comparator threshold voltage, this does not affect the energy harvesting and makes the "hard" active converter a competitive solution with respect to the passive one. 

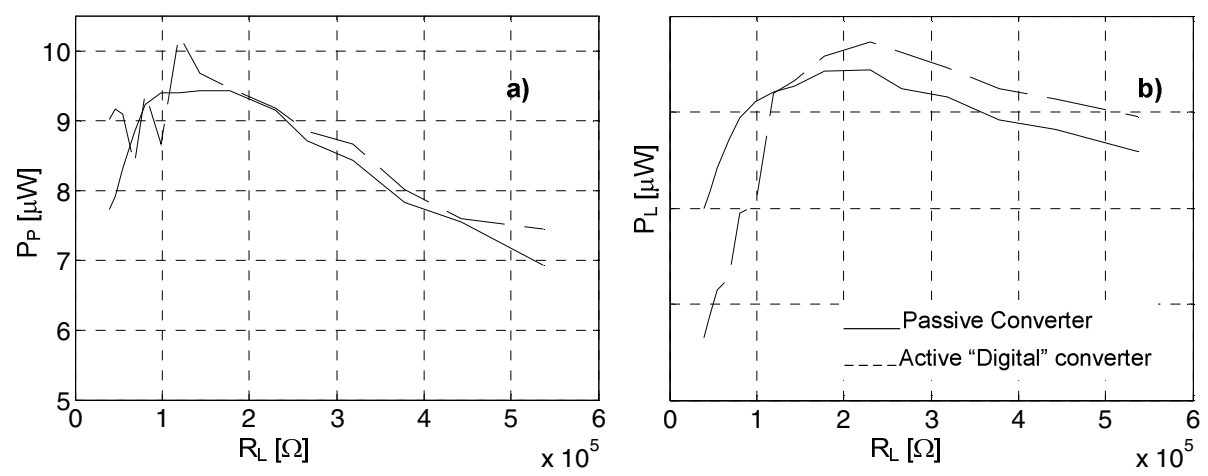

Fig. 14. a, b) Experimental results about input and output powers $\left(\mathrm{P}_{\mathrm{P}}\right.$ and $\mathrm{P}_{\mathrm{L}}$ ) respectively. Comparison between active "digital" converter and passive converter, this was realized with Schottky BAT86 diodes.

The performances of the "soft" solutions were evaluated at different values of the mechanical acceleration so to obtain different values of $\mathrm{V}_{\mathrm{PO}}$ (i.e., 1.5, 2 and $2.5 \mathrm{~V}$ ); Figure 15 a, b show the obtained results, comparing them with the corresponding behaviour of the passive converter, as usual. It is possible to see that under any condition the "soft" converter draws a lower power from the piezoelectric transducer than the passive one. On an absolute scale this means that the combination of the piezoelectric transducer and the "soft" converter makes a less effective energy harvester.

Returning to the interpretation of the experimental results the "soft" converter is generally less capable of extracting energy from the transducer with respect to both the "hard" rectifier and the passive one.

At this point one should expect that also the power delivered to the load resistance $\mathrm{P}_{\mathrm{L}}$ is lower, but the experimental results of Figure 15 show that the two rectifiers are equivalent when $\mathrm{V}_{\mathrm{PO}}$ is $1.5 \mathrm{~V}$, that the active rectifier performs better at lower load resistance values for $\mathrm{V}_{\mathrm{PO}}=2$, and that finally it outperforms the diode converter on a wide load range for $\mathrm{V}_{\mathrm{PO}}=2.5 \mathrm{~V}$.

This means that the "soft" converter has an efficiency higher than the passive one and its advantage increases as $\mathrm{V}_{\mathrm{PO}}$ is higher. The reason is that the power consumption of the driving circuitry weights most at lower values of $\mathrm{P}_{\mathrm{P}}$, that is at the lower values of input voltage.

Coming to a comparison between the two proposed converters, when the threshold voltages have the correct sign, the performances of the "hard" solution (ref. Figure 14b) are better with respect to both "soft" and passive converter.

Efficiency $(\eta)$ of an energy conversion system is defined as the ratio between its output power with respect to its input power. This is usually used as figure of merit which allows to compare different solutions which work at the same output power; this means that the system which requires the lower input power is the better one, under the hypothesis that the input source could be able to supply an infinite input power. 

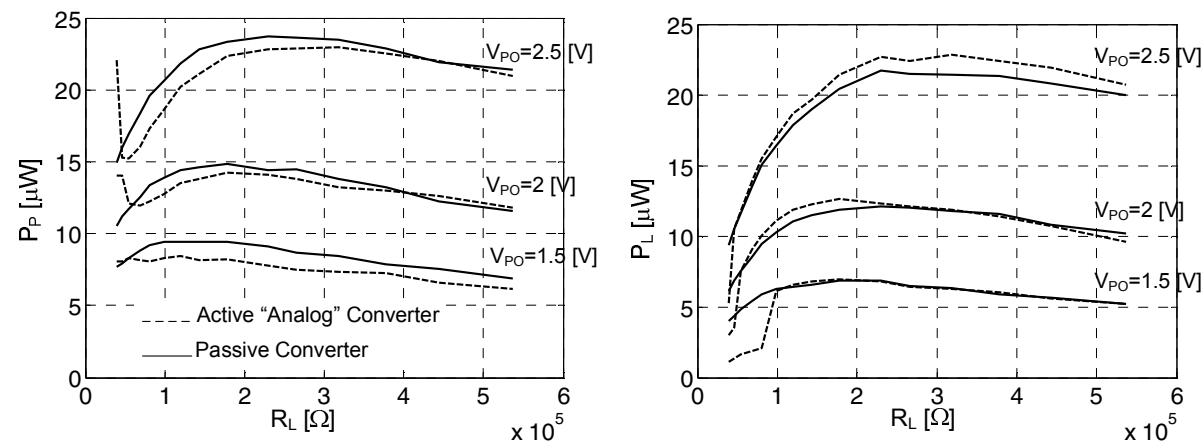

Fig. 15. Measured input $\left(\mathrm{P}_{\mathrm{P}}\right)$ and output $\left(\mathrm{P}_{\mathrm{L}}\right)$ powers at different values of $\mathrm{V}_{\mathrm{PO}}$, comparison between passive Schottky based converter and "analog" AC-DC converter

Comparing the experimental results obtained at $\mathrm{V}_{\mathrm{PO}}=1.5 \mathrm{~V}$ (see Figure 16) for the three different AC-DC converters it is possible to see that their $\eta$ can not be used as figure of merit to evaluate the performances of the energy harvesting system. In fact, the "soft" converter shows the better performance in terms of efficiency with respect any other solution, but it is equivalent at the passive one in terms of output power. Furthermore, "hard" solution outperforms any other converter in terms of output power. This is due to the fact that the input energy source, that is the piezoelectric transducer, is not an infinite energy source and its matching with the front-end circuitry is an important aspect which makes effectiveness the energy harvesting system. Furthermore, none of the systems is controlling the output voltage, hence the output power is not fixed, but it is the natural result of the behaviour of the entire system. All these considerations give strength to the choice of focusing on a different figure of merit (FoM) to compare the converters; for example this could be the ratio of the harvested power with respect to the maximum power theoretically available from the transducer. This last quantity can be defined studying the behaviour of a piezoelectric transducer, at no load condition, when exposed to a sinusoidal acceleration at frequency $f$ which strains the transducer as shown in Figure 17.

Starting from its rest condition at $t_{0}$, that is no strain and no electrical charges on its plates, the cantilever reaches its maximum positive bending (and strain) at $t_{1}$. At this point the collected electrical charge is $\mathrm{Q}_{\mathrm{P}}$ and the piezoelectric output voltage is equal to $\mathrm{V}_{\mathrm{PO}}$. If an ideal front-end circuitry could recover all of this charge, completely discharging the transducer, when the cantilever reaches its maximum negative deflection at $t_{3}$ it will collect a negative charge two times higher $\left(-2 Q_{P}\right)$ because the deflection is doubled; in fact, at $t_{2}$ the collected charge is already equal to - $Q_{p}$. As a consequence the piezoelectric peak voltage at $t_{3}$ is equal to $-2 V_{P O}$ and the transduced energy is:

$$
E_{t_{1}-t_{3}}=\frac{1}{2} C_{P}\left(-2 V_{P O}\right)^{2}=2 C_{P} V_{P O}^{2}
$$

Now imagine that at $t_{3}$, again ideally, capacitor $C_{P}$ is newly, fully discharged while, at the same time, the associated energy is stored. Since the excursion in the $t_{3}-t_{4}$ interval 
starts with no charge on the capacitor plates, it evolves as a replica of what happened in the $t_{1}-t_{3}$ interval, only the sign of the voltage is inverted. This means that the available energy at $t_{4}$ is equal to:

$$
E_{t_{3}-t_{4}}=\frac{1}{2} C_{P}\left(2 V_{P O}\right)^{2}=2 C_{P} V_{P O}^{2}
$$

From this moment on the phenomenon continues to repeat itself with a periodicity equal to $\mathrm{t}_{4}-\mathrm{t}_{1}$.

Summarizing the total energy that can be harvested during one cycle becomes:

$$
E_{\text {cycle }}=E_{t_{1}-t_{3}}+E_{t_{3}-t_{4}}=4 C_{P} V_{P O}^{2}
$$

Finally, the maximum power theoretically available from the transducer is:

$$
P_{\max }=4 C_{P} V_{P O}^{2} f
$$

The quantity expressed by (9) will be used to weight the power actually delivered to the load by the proposed rectifiers. This approach allows to compare the various circuits, but is also allows to quantify how effective a given circuit is in harvesting the power made available by the transducer.

The figure of merit, which is function of $\mathrm{R}_{\mathrm{L}}$, can be defined as:

$$
\text { F.o.M. }=\frac{P_{L}}{P_{\max }}
$$

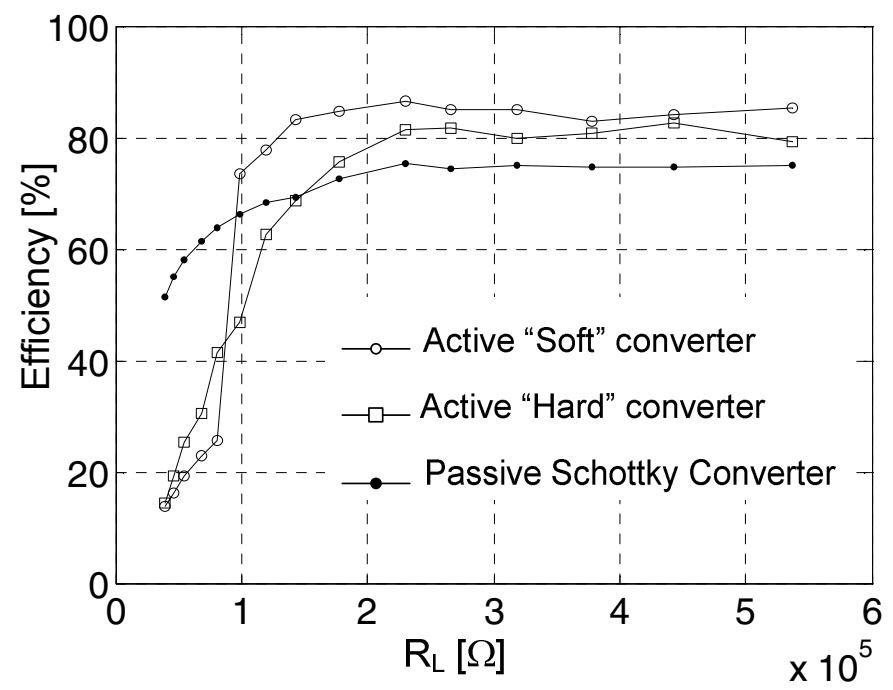

Fig. 16. Efficiency of the AC-DC converters at $\mathrm{V}_{\mathrm{PO}}=1.5 \mathrm{~V}$ against load resistance 


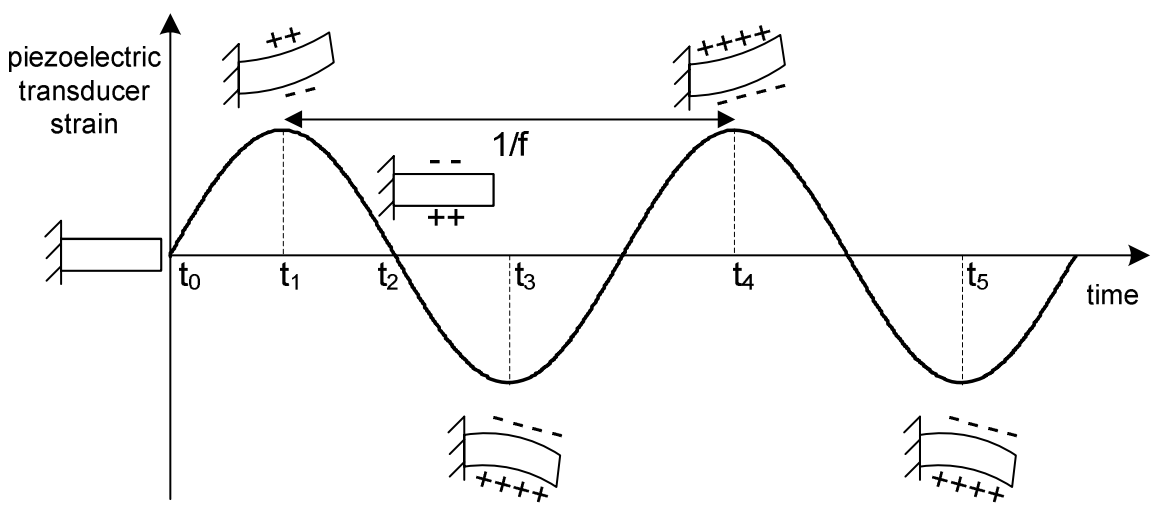

Fig. 17. Mechanical strain of piezoelectric trasnducer when it is excited by a sinusoidal mechanical acceleration

Figure 18 shows this figure of merit for the presented AC-DC converters in case of $\mathrm{V}_{\mathrm{PO}}$ equal to $1.5 \mathrm{~V}$. It is possible to see that "hard" topology outperforms both "soft" and passive ones but, at the same time, none of the converters is able to harvest all the power the transducer could deliver.

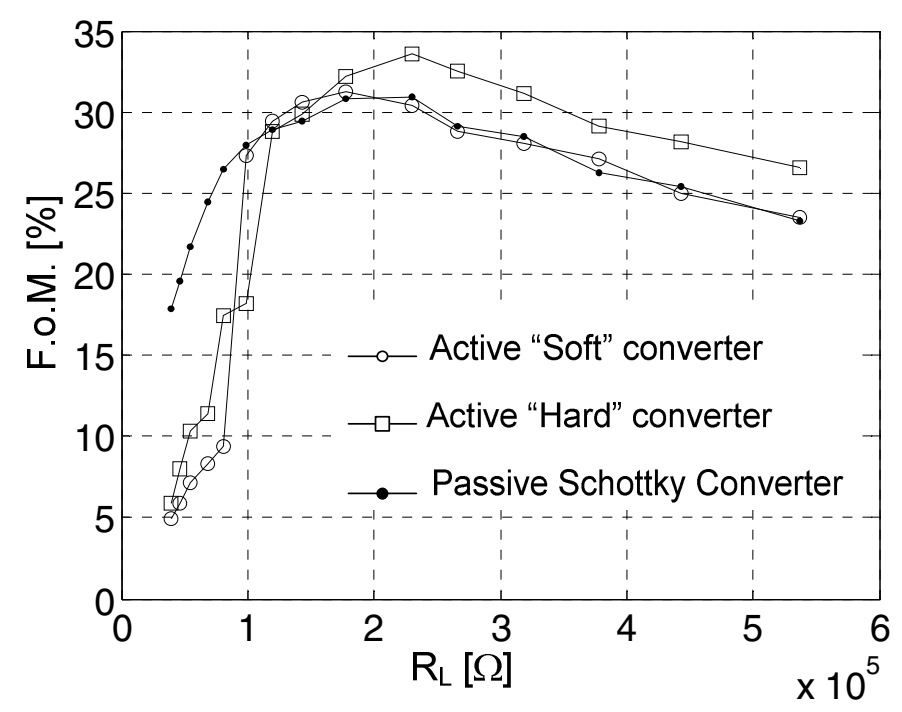

Fig. 18. Power harvested by the AC-DC converters with respect to the maximum defined in Equation 7

\section{Conclusions}

This paper presents two actives AC-DC converters which can be used into piezoelectric energy scavenging systems. The basic circuit is the voltage doubler 
topology, where the switches are actively driven. The two considered driving circuitries are realized with comparators and operational amplifier respectively.

It was shown that the first configuration, called "hard" is slightly more difficult to be designed than the second one, called "soft". In particular the comparators need to have an hysteretic characteristic and if the value of the thresholds is different from the ideal one the efficiency of the converter could be significantly deteriorated. Hence a lot of effort is needed to minimize the process dependence of the thresholds. On the contrary, the "soft" configuration drives the switches with a regulation loop approach, which is far less sensitive to process uncertainties.

A test chip has been diffused in STMicroelectronics 5V CMOS technology and experimental results are presented. The tests were realized using a piezoelectric transducer working in 31-mode, excited by a electro-dynamic shaker.

The performances of the proposed active solutions were evaluated at different values of a load resistance and they were compared with the ones of a passive Schottky-based voltage doubler. The average power supplied by the piezoelectric transducer and the power delivered on a load resistance were measured. The experimental results show that, under the same mechanical excitation, the "hard" solution outperforms the other two converters in terms of power delivered to the resistive load. The "soft" solution was less effective because the delivered average power by the piezoelectric transducer was lower; this was probably due to a different matching between the piezoelectric transducer and the converter itself which is better in the "hard solution". The efficiencies of the three converters were compared at different load conditions. The "soft" solution, despite its lower effectiveness, has the higher efficiency values with respect to the other two converters: this means that the converter efficiency can not be used as the only figure of merit for an energy harvesting system, it is necessary to evaluate also the effectiveness of the converter in terms of average power harvested from the transducer and delivered to the load.

To do this a dedicated figure of merit was introduced which compares the output power with the power that the transducer can theoretically deliver rather than with the power it actually delivers.

\section{References}

[1] Roundy, S.: Energy Scavenging for Wireless Sensor Nodes with a focus on Vibration to Electricity Conversion. PhD Thesis, The University of California Berkeley (Spring 2003)

[2] Roundy, S., et al.: Improving Power Output for Vibration Based Energy Scavengers. Pervasive Computing, 28-36 (January/March 2005); Published by the IEEE and IEEE ComSoc.

[3] Le Jifeng Han, T.T., von Jouanne, A., Mayaram, K., Fiez, T.S.: Piezoelectric Micro Power Generation Interface Circuits. IEEE Journal of Solid State Circuits 41(6), 14111420 (2006)

[4] Dallago, E., Frattini, G., Miatton, D., Ricotti, G., Venchi, G.: Self Supplied Integrable High Efficiency AC-DC Converter for Piezoelectric Energy Scavenging Systems. In: International Sympoium on Circuits and Systems, ISCAS 2007, New Orleans, LO, May 27-30, pp. 1633-1636 (2007) 
[5] Dong, Z., Allen, P.E.: Low Voltage, Supply Independent CMOS Bias Circuit. In: The 2002 45th Midwest Symposium on Circuits and Systems, August 4-7, vol. 3, pp. 568-570 (2002)

[6] Peano, F., Tambosso, T.: Design and Optimization of a MEMS Electret Based Capacitive Energy Scavenger. Journal of Microelectromechanical Systems 14(3), 429-435 (2005)

[7] Renaud, M., Sterken, T., Fiorini, P., Puers, R., Baert, K., van Hoof, C.: Scavenging Energy from Human Body, Design of a Piezoelectric Transducer. In: The 13th International Conference on Solid State Sensors, Actuators and Mycrosystems, Seoul, Korea, June 5-9, pp. 784-787 (2005)

[8] Stark, I.: Thermal Energy Harvesting with Thermo Life®. In: Proceedings of the International Workshop on Wearable and Implantable Body Sensor Networks, BSN 2006, pp. 19-22. IEEE Computer Society, Los Alamitos (2006)

[9] Han, J., von Jouanne, A., Le, T., Mayaram, K., Fiez, T.S.: Novel power conditioning Circuits for Piezoelectric Micro Power Generators. In: APEC 2004, February 2004, vol. 3, pp. 1541-1546 (2004)

[10] Sterken, T., Fiorini, P., Baert, K., Puers, R., Borghs, G.: An Electret-Based Electrostatic $\mu$-generator. In: The 12th IEEE International Conference on Solid State Sensors, Actuators and Microsystems, Boston, June 8-12, vol. 2, pp. 1291-1294 (2003)

[11] Dallago, E., Frattini, G., Miatton, D., Ricotti, G., Venchi, G.: Integrable High Efficiency AC-DC converter for Piezoelectric Energy Scavenging System. In: IEEE International Conference on Portable Information Devices, Orlando (FL), USA, March 25-29, pp. 1-5 (2007) 